\title{
Early Dark Energy at High Redshifts: Status and Perspectives
}

\author{
Jun-Qing Xia周 and Matteo Viel ${ }^{2,3}$ \\ ${ }^{1}$ Scuola Internazionale Superiore di Studi Avanzati, Via Beirut 2-4, I-34014 Trieste, Italy \\ ${ }^{2}$ INAF-Osservatorio Astronomico di Trieste, Via G.B. Tiepolo 11, I-34131 Trieste, Italy and \\ ${ }^{3}$ INFN/National Institute for Nuclear Physics, Via Valerio 2, I-34127 Trieste, Italy
}

(Dated: October 29, 2018)

\begin{abstract}
Early dark energy models, for which the contribution to the dark energy density at high redshifts is not negligible, influence the growth of cosmic structures and could leave observable signatures that are different from the standard cosmological constant cold dark matter $(\Lambda \mathrm{CDM})$ model. In this paper, we present updated constraints on early dark energy using geometrical and dynamical probes. From WMAP five-year data, baryon acoustic oscillations and type Ia supernovae luminosity distances, we obtain an upper limit of the dark energy density at the last scattering surface (lss), $\Omega_{\mathrm{EDE}}\left(z_{\mathrm{lss}}\right)<2.3 \times 10^{-2}(95 \%$ C.L.). When we include higher redshift observational probes, such as measurements of the linear growth factors, Gamma-Ray Bursts (GRBs) and Lyman- $\alpha$ forest (Ly $\alpha$ ), this limit improves significantly and becomes $\Omega_{\mathrm{EDE}}\left(z_{\mathrm{lss}}\right)<1.4 \times 10^{-3}$ (95\% C.L.). Furthermore, we find that future measurements, based on the Alcock-Paczyński test using the $21 \mathrm{~cm}$ neutral hydrogen line, on GRBs and on the Ly $\alpha$ forest, could constrain the behavior of the dark energy component and distinguish at a high confidence level between early dark energy models and pure $\Lambda$ CDM. In this case, the constraints on the amount of early dark energy at the last scattering surface improve by a factor ten, when compared to present constraints. We also discuss the impact on the parameter $\gamma$, the growth rate index, which describes the growth of structures in standard and in modified gravity models.
\end{abstract}

\section{INTRODUCTION}

Current cosmological observations, such as the cosmic microwave background (CMB) measurements of temperature anisotropies and polarization [1] and the redshiftdistance measurements of Type Ia Supernovae (SNIa) at $z<2$ 2], have demonstrated that the Universe is now undergoing an accelerated phase of expansion and that its total energy budget is dominated by the dark energy component. The nature of dark energy is one of the biggest unsolved problems in modern physics and has been extensively investigated in recent years, both under the theoretical and the observational point of view. The simplest candidate of dark energy is the cosmological constant $(\Lambda \mathrm{CDM})$ : however, this model suffers of the fine-tuning and coincidence problems [3]. To lift these tensions, many alternative dynamical dark energy models, such as quintessence [4], phantom [5], K-essence [6] and quintom [7], have been proposed (for a review see Ref. [8]).

Among all the dynamical dark energy models, we will focus here on early dark energy (EDE) ones, in which a small fraction of dark energy is present up to the last scattering surface (lss), unlike $\Lambda \mathrm{CDM}$ for which $\Omega_{\mathrm{DE}}\left(z_{\mathrm{lss}}\right) \simeq 0$, The differences between early dark energy models and pure $\Lambda \mathrm{CDM}$ are particularly evident at high redshifts, over a large fraction of the cosmic time, when the first structures form. EDE has been shown to influence the growth of cosmic structures (both in the linear and in the non-linear regime), to change the age

\footnotetext{
*Electronic address: xia@sissa.it

${ }^{\dagger}$ Electronic address: viel@oats.inaf.it
}

of the universe, to have an influence on CMB physics, to impact on the reionization history of the universe, to modify the statistics of giant arcs in strong cluster lensing statistics [9, 10, 11, 12, 13, 14, 15, 16, 17].

The reason for addressing this particular model of dark energy is also driven by the increasing availability of high redshift observations that somewhat bridge the gap between the CMB and very local cosmological probes. Dark energy studies rely mainly on: the clustering properties of luminous red galaxies 18], baryonic acoustic oscillations (BAO) [19, 20], weak lensing data 21, 22, high redshift SNIa 23], the Ly $\alpha$ forest [24, 25], GRBs 26, 27] as standard candles, and also the number of high redshift galaxies and clusters of galaxies [28] that could be studied with deep field observations. Of course, since the present data sets seem to be in good agreement with pure $\Lambda \mathrm{CDM}$ model, we hope that these observations could open up a new high redshift window on the properties of dark energy and possible allow to confirm or disproof its redshift evolution. Such a difficult measurement would have profound implications for physical cosmology and particle physics.

In this paper we extend our studies of dark energy behavior into the redshift range $2<z<1100$, which covers the so-called dark ages, and present constraints on EDE from current and future cosmological observations. We will use CMB, BAO, SNIa, Ly $\alpha$ forest observations of growth factors and matter power spectrum and the GRBs from current available data sets, while for forecasts we will add to Planck-like observations, some higher redshift GRBs, some improved Ly $\alpha$ constraints and a measurement that could be potentially very important of the so-called Alcock-Paczyński (AP, [29]) test using $21 \mathrm{~cm}$ maps [30, 31]. As we will see, the particular parameterization of EDE chosen will be such that 
the higher the redshift the tighter the constraints will be on the parameters describing the given model. Thereby, futuristic intergalactic medium $\operatorname{Ly} \alpha$ forest data, GRBs distance moduli and the $21 \mathrm{~cm}$ maps, before or around reionization, are expected to be very promising probes of dynamical dark energy models (see also Ref.[13] for the impact of these models on the reionization history of the universe). We note that the behavior of dark energy in this redshift range has been exploited by Ref. 32], who constrained the EDE density of to be less than $2 \%$ of the total energy density. Here, we will improve this limit by adding several different observations: as mentioned before this is not only important to get stronger constraints on some cosmological parameters but also to understand the consistency of different probes and how systematic effects impact on the final derived measurements (e.g. Refs. 33, 34]). Of course, we stress that while our approach has the great advantage of using dynamical and geometrical cosmological probes in the linear or quasilinear regime, other approaches, based for example on haloes concentrations, could also be envisaged and must rely on an accurate comparison with numerical simulations [16].

Our paper is organized as follows: in Sec. II we describe the theoretical framework of the early dark energy model and the datasets we used. Sec. III contains our main global fitting results from the current observations. In Sec. IV we present the forecasts for the future measurements while Sec. $\mathrm{V}$ is dedicated to the conclusions and discussion.

\section{METHOD AND DATA}

\section{A. Parameterization of early dark energy}

We decided to use the mocker model introduced in Ref. 32] motivated by the following two observational facts: i) the best-fit model is pure $\Lambda$ CDM model and the amount of dark energy at the last scattering surface is constrained to be close to zero from $\mathrm{CMB}$ and big bang nucleosynthesis (BBN) observations; ii) an equation of state of the dark energy component which is rapidly evolving (i.e. $d w / d z \gg 1$ ) seems to be ruled out by highredshift SNIa [23].

For these EDE models the parameterization reads:

$$
w_{\mathrm{EDE}}(a)=-1+\left[1-\frac{w_{0}}{1+w_{0}} a^{C}\right]^{-1},
$$

where $a=1 /(1+z)$ is the scale factor, $w_{0}$ is the present equation-of-state of dark energy and $C$ characterizes the "running" of the equation of state. Consequently, the evolution of dark energy density can easily be obtained via energy conservation as:

$$
\frac{\rho_{\mathrm{EDE}}(a)}{\rho_{\mathrm{EDE}}(1)}=\left[\left(1+w_{0}\right) a^{-C}-w_{0}\right]^{3 / C} .
$$

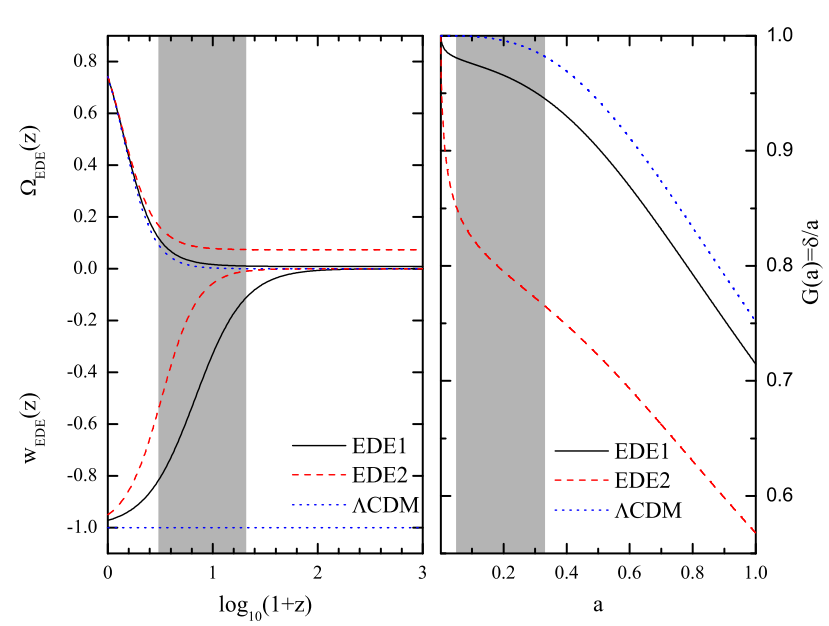

S

Figure 1: Left panel: the evolution of dark energy density and equation of state for three models, $w_{0}=-0.972$ and $C=$ 1.858 (EDE1), $w_{0}=-0.95$ and $C=2.5$ (EDE2) and pure $\Lambda$ CDM. Right panel: the linear growth factor $G(a)=\delta(a) / a$ normalized at $z=\infty$ for the three models. The shaded area represents the redshifts that we will be mainly probing in the present work $(z \sim 2-20)$.

In Fig. 1 we plot the dark energy density (upper part of the left panel) and equation of state (bottom part of the left panel) as a function of redshift for three different models: pure $\Lambda$ CDM (dotted blue line), EDE1 (solid black line) which has $\left(w_{0}, C\right)=(-0.972,1.858)$ and EDE2 (dashed red line) which has $\left(w_{0}, C\right)=(-0.95,2.5)$. All these models fit the CMB and the lower redshift SNIa constraints very well. In the right panel, we also show the linear growth rate (growth factors divided by the scale factor) normalized at $z=\infty$ and compare the different evolutions with redshifts in the three cases: differences of the order of $20 \%$ are visible at $z \sim 3$. In the two panels we also show as a shaded vertical band the region in the redshift range $z=2-20$, a period which is roughly $25 \%$ of the age of the universe. This is the redshift range we will be (mainly) focussing on in the rest of the paper in order to discriminate between the different dark energy models.

We stress that this is just one of the possible parameterizations for early dark energy models, another one has been suggested by Ref. [35] and recently used in Ref. [17]. However, we prefer to use the mocker model in order to compare with the findings of Ref. 32 and because this parameterization has a smooth redshift derivative at low $z$ for $w(z)$. We note that one of the most important parameters is the amount of dark energy during the structure formation period and this is given by $\left(a_{\mathrm{eq}}\right.$ is the matter-radiation equality scale factor):

$$
\Omega_{\mathrm{EDE}, \mathrm{sf}}=-\left(\ln a_{\mathrm{eq}}\right)^{-1} \int_{\ln a_{\mathrm{eq}}}^{0} \Omega_{\mathrm{EDE}}(a) \mathrm{d} \ln a,
$$


and we will also quote this value in the rest of the paper in order to compare with other works and constraints as well (e.g. Refs. 9, 15]).

We will also use some growth factors measurements obtained mainly through Ly $\alpha$ forest observations and one lower redshift from Sloan Digital Sky Survey (SDSS) galaxies. For this purpose we will compute the growth factors for the EDE models with a subroutine implemented in the publicly available Monte Carlo Markov Chains (MCMC) package CosmoMC [36] that solves the second order differential equations for the growth factor as in Ref.[10]. Furthermore, we also modify CosmoMC to include the perturbations of dynamical dark energy models generally as done in Refs. [37].

\section{B. Current and Future Datasets}

\section{Current Observations}

We will rely here on the following cosmological probes: i) CMB anisotropies and polarization; ii) baryonic acoustic oscillations in the galaxy power spectra; iii) SNIa distance moduli; iv) GRBs distance moduli; v) the Ly $\alpha$ forest growth factors and matter power spectrum measurements.

In the computation of CMB power spectra we have included the WMAP five-year (WMAP5) temperature and polarization power spectra with the routines for computing the likelihood supplied by the WMAP team [38]. Besides the WMAP5 information, we also use some distance-scale indicators.

BAOs have been detected in the current galaxy redshift survey data from the SDSS and the Two-degree Field Galaxy Redshift Survey (2dFGRS) [19, 20, 39, 40]. The $\mathrm{BAO}$ can directly measure not only the angular diameter distance, $D_{A}(z)$, but also the expansion rate of the universe, $H(z)$, which is powerful for studying dark energy [41]. Since current BAO data are not accurate enough for extracting the information of $D_{A}(z)$ and $H(z)$ separately [42], one can only determine an effective distance [19]:

$$
D_{v}(z) \equiv\left[(1+z)^{2} D_{A}^{2}(z) \frac{c z}{H(z)}\right]^{1 / 3}
$$

In this paper we use the Gaussian priors on the distance ratios $r_{s}\left(z_{d}\right) / D_{v}(z)$ :

$$
\begin{aligned}
& r_{s}\left(z_{d}\right) / D_{v}(z=0.20)=0.1980 \pm 0.0058 \\
& r_{s}\left(z_{d}\right) / D_{v}(z=0.35)=0.1094 \pm 0.0033
\end{aligned}
$$

with a correlation coefficient of 0.39 , extracted from the SDSS and 2dFGRS surveys [40], where $r_{s}$ is the comoving sound horizon size and $z_{d}$ is drag epoch at which baryons were released from photons given by Ref. 43].

The SNIa data provide the luminosity distance as a function of redshift which is also a very powerful measurement of dark energy evolution. The supernovae data we use in this paper are the recently released Union compilation (307 samples) from the Supernova Cosmology project [2], which include the recent samples of SNIa from the (Supernovae Legacy Survey) SNLS and ESSENCE survey, as well as some older data sets, and span the redshift range $0 \lesssim z \lesssim 1.55$. In the calculation of the likelihood from SNIa we have marginalized over the nuisance parameter as done in [44] and ignored the systematic errors to improve our results ${ }^{1}$.

Furthermore, we make use of the Hubble Space Telescope (HST) measurement of the Hubble parameter $H_{0} \equiv 100 h \mathrm{~km} \mathrm{~s}^{-1} \mathrm{Mpc}^{-1}$ by a Gaussian likelihood function centered around $h=0.72$ and with a standard deviation $\sigma=0.08$ [46].

In order to constrain the early dark energy model at high redshifts, we also include the GRBs, linear growth factors and Ly $\alpha$ forest data.

GRBs can potentially be used to measure the luminosity distance out to higher redshift than SNIa. Recently, several empirical correlations between GRB observables were reported, and these findings have triggered intensive studies on the possibility of using GRBs as cosmological "standard" candles. However, due to the lack of low-redshift long GRBs data to calibrate these relations, in a cosmology-independent way, the parameters of the reported correlations are given assuming an input cosmology and obviously depend on the same cosmological parameters that we would like to constrain. Thus, applying such relations to constrain cosmological parameters leads to biased results. In Ref. 47 the circular problem is naturally eliminated by marginalizing over the free parameters involved in the correlations; in addition, some results show that these correlations do not change significantly for a wide range of cosmological parameters [48]. Therefore, in this paper we use the 69 GRBs sample over a redshift range from $z=0.17-6.60$ published in Ref. [26] but we keep in mind the issues related to the "circular problem" that are more extensively discussed in Ref. 47.

In Table I we list two types of linear growth factors data we use: i) the normalization $\sigma_{8}$ inferred from the SDSS Ly $\alpha$ power spectrum ${ }^{2}$ [49, 50]; ii) the linear growth rate $f \equiv \Omega_{\mathrm{m}}^{\gamma}$ from galaxy power spectrum at low redshift 51] and Ly $\alpha$ growth factor measurement obtained from

\footnotetext{
${ }^{1}$ In this paper, we find that the low redshifts probes could not constrain on the early dark energy models very well. Therefore, we focus on the constraints on the EDE model from high redshifts probes. Furthermore, in Ref. 45] we discussed the effect of constraints on dark energy models with or without systematic error. When including the systematic error, the constraints are slightly weaker, but the main results are not changed.

2 These data at different redshifts are not totally independent. The derived $\sigma_{8}$ values are obtained by using the covariance matrix as measured from the data of the SDSS flux power spectrum and there are (very weak) correlations in the flux power between different redshift bins. So, although not totally independent, we regard the derived values of the power spectrum amplitude from the SDSS flux power as nearly independent.
} 
TABLE I. $\sigma_{8}$ values and growth rates with $1 \sigma$ error bars from which we derived the linear growth factors used in our analysis.

\begin{tabular}{cccc}
\hline$z$ & $\sigma_{8}$ & $\sigma_{\sigma_{8}}$ & Ref. \\
\hline 2.125 & 0.95 & 0.17 & {$[49]$} \\
2.72 & 0.92 & 0.17 & \\
\hline 2.2 & 0.92 & 0.16 & {$[50]$} \\
2.4 & 0.89 & 0.11 & \\
2.6 & 0.98 & 0.13 & \\
2.8 & 1.02 & 0.09 & \\
3.0 & 0.94 & 0.08 & \\
3.2 & 0.88 & 0.09 & \\
3.4 & 0.87 & 0.12 & \\
3.6 & 0.95 & 0.16 & \\
3.8 & 0.90 & 0.17 & \\
\hline$z$ & $f$ & $\sigma_{f}$ & Ref. \\
\hline 0.15 & 0.51 & 0.11 & {$[51]$} \\
3.00 & 1.46 & 0.29 & {$[25]$} \\
\hline
\end{tabular}

the SDSS Ly $\alpha$ power spectrum at $z=3$ by Ref. 25$]$.

We have used two Ly $\alpha$ forest data sets: i) the high resolution QSO absorption spectra presented in Ref.[49] consisting of the LUQAS sample [52] and the reanalyzed data in Ref.[53] (C02, this data set as a whole will be labelled as VHS in the following); ii) the SDSS Ly $\alpha$ forest sample presented in Ref. [54]. The SDSS Ly $\alpha$ forest data set consists of 3035 QSO spectra with low resolution $(R \sim 2000)$ and low $\mathrm{S} / \mathrm{N}(<10$ per pixel $)$ spanning a wide range of redshifts $(z=2.2-4.2)$, while the LUQAS and the C02 samples contain 57 high resolution $(R \sim 45000)$, high signal-to-noise ( $>50$ per pixel) QSO spectra with median redshifts of $z=2.125$ and $z=2.72$, respectively. The flux power spectrum of the Ly $\alpha$ forest is the quantity which is observed and needs to be modeled at the percent or sub-percent level using accurate numerical simulations that incorporate the relevant cosmological and astrophysical processes, in order to extract the underlying (linear) dark matter power spectrum. In this paper, we will use the derived linear power spectrum measured by the data set of VHS, based on "VHSLy $\alpha$ " together with the SDSS Ly $\alpha$ power spectrum "SDSSLy $\alpha$ ", since they are in agreement and this latter has a stronger constraining power. More precisely, the VHSLy $\alpha$ power spectrum consists of estimates of the linear dark matter power spectrum at nine values in the wavenumber space $k$ at $z=2.125$ and nine values at $z=2.72$, in the range $0.003<k(\mathrm{~s} / \mathrm{km})<0.03$, while the SDSSLy $\alpha$ consists of a single measurement at $z=3$ and $k=0.009 \mathrm{~s} / \mathrm{km}$ of amplitude, slope and curvature. The estimate of the uncertainty of the overall amplitude of the matter power spectrum is $29 \%$ for the first dataset and $\sim 14 \%$ for the second. This estimate takes into account possible systematic and statistical errors (see the relevant tables of VHS for a detailed discussion). The code assigns a Gaussian prior to the corresponding nuisance parameter and marginalize over it. Finally, we will also rely on the growth factor measurements from the
TABLE II. Assumed experimental specifications for the mock Planck-like measurements. The noise parameters $\Delta_{T}$ and $\Delta_{P}$ are given in units of $\mu \mathrm{K}$-arcmin.

\begin{tabular}{cccccc}
\hline \hline$f_{\text {sky }}$ & $l_{\max }$ & $(\mathrm{GHz})$ & $\theta_{\text {fwhm }}$ & $\Delta_{T}$ & $\Delta_{P}$ \\
\hline 0.65 & 2500 & 100 & $9.5^{\prime}$ & 6.8 & 10.9 \\
& & 143 & $7.1^{\prime}$ & 6.0 & 11.4 \\
& & 217 & 5.0 & 13.1 & 26.7 \\
\hline \hline
\end{tabular}

two data sets, derived using numerical simulations of the observed flux power, and we will label this as GFLy $\alpha$.

\section{Future Measurements}

In order to forecast future measurements we will use the same observables as before without $\mathrm{BAO}$ but exploiting the role of $21 \mathrm{~cm}$ maps at high redshifts.

For the simulation with PLANCK [55], we follow the method given in Ref. [56] and mock the CMB temperature (TT) and polarization (EE) power spectra and temperature-polarization cross correlation (TE) by assuming a given fiducial cosmological model. In Table II, we list the assumed experimental specifications of the future (mock) Planck measurement.

The proposed satellite SNAP (Supernova / Acceleration Probe) will be a space based telescope with a one square degree field of view that will survey the whole sky [57]. It aims at increasing the discovery rate of SNIa to about 2000 per year in the redshift range $0.2<z<1.7$. In this paper we simulate about 2000 SNIa according to the forecast distribution of the SNAP [58]. For the error, we follow the Ref.[58] which takes the magnitude dispersion to be 0.15 and the systematic error $\sigma_{\mathrm{sys}}=0.02 \times z / 1.7$. The whole error for each data is given by $\sigma_{\mathrm{mag}}\left(z_{i}\right)=\sqrt{\sigma_{\mathrm{sys}}^{2}\left(z_{i}\right)+0.15^{2} / n_{i}}$, where $n_{i}$ is the number of supernovae of the $i^{\prime}$ th redshift bin. Furthermore, we add as an external data set a mock set of 400 GRBs, that mimic SWIFT [60] observations, in the redshift range $0<z<6.4$ with an intrinsic dispersion in the distance modulus of $\sigma_{\mu}=0.16$ and with a redshift distribution very similar to that of Figure 1 of Ref. [59].

For the linear growth factors data, we simulate the mock data from the fiducial model of Table I with the error bars reduced by a factor of two. This is probably reasonable given the larger amounts of $\mathrm{Ly} \alpha$ forest data (e.g. SDSS-III or the X-shooter spectrograph 61]) that will become available soon as long with a better control of several systematics errors and more importantly on the thermal history of the intergalactic medium.

We also simulate the Ly $\alpha$ power spectrum, consisting of three data points at $z=2,3$ and 3.5 and at wavenumbers of $0.002 \mathrm{~s} / \mathrm{km}, 0.009 \mathrm{~s} / \mathrm{km}$ and $0.02 \mathrm{~s} / \mathrm{km}$, with $1 \%$ fractional errors in a similar way as done by Ref. 62], in which a forecasting on the perspective of constraining the neutrino masses was presented. 
The availability of $21 \mathrm{~cm}(1420 \mathrm{MHz})$ maps with future experiments like LOFAR 63] will allow to measure in a given frequency interval and for a given position in the sky the differential brightness temperature of a patch of gas against the CMB. Thus a completely new window on the neutral hydrogen content of the universe, regarded as a tracer of cosmic structures and of astrophysical processes, will be opened soon. The AP test 29] assumes that the for an isotropically distributed set of astronomical objects the separations along and across the line-of-sights scale differently and thus generate anisotropies. The geometrical distortion depends on the quantity $\left[H(z) D_{A}(z)\right]^{-1}$ that thereby can be measured and provide constraints on some parameters. The extended LOFAR will operate between 30 and 240 $\mathrm{MHz}$, with spatial resolution of the order of arcseconds, probing the universe in the redshift range $z \sim 6-45$. For our purposes here we will assume that the quantity $\left[H(z) D_{A}(z)\right]^{-1}$, will be measured at $z=6.5,8,12$ with $5 \%$ precision. Although other measurements could be performed using $21 \mathrm{~cm}$ datasets, such as (quite optimistically) a full measurement of the matter power spectrum at very high redshifts, we will concentrate here only on the AP test, since this appears to be easier to be performed and it is less sensitive to the astrophysical uncertainties that relate the differential brightness temperature to the matter density (see however Ref. 64 for other possible and very promising constraints). It is nevertheless important to note that LOFAR sensitivity drops for $z>12$ and useful HI maps at these redshifts could only be obtained with future ground based telescopes like SKA [65] and with a better understanding of the foregrounds that contaminate the cosmological signal (see for example the discussion in Ref. [30]).

Here, we will use a somewhat more conservative approach and simulate a measurement of the quantity described above in a redshift range accessible to the LOFAR experiment. Although challenging, we believe that the AP test could be performed and give constraints similar to those obtained here.

\section{RESULTS FROM PRESENT DATASETS}

In our analysis, we perform a global fitting using the CosmoMC package. We assume purely adiabatic initial conditions and a flat universe, with no tensor contribution. We vary the following cosmological parameters with top-hat priors: the dark matter energy density $\Omega_{c} h^{2} \in[0.01,0.99]$, the baryon energy density $\Omega_{b} h^{2} \in$ $[0.005,0.1]$, the primordial spectral index $n_{s} \in[0.5,1.5]$, the primordial amplitude $\log \left[10^{10} A_{s}\right] \in[2.7,4.0]$ and the angular diameter of the sound horizon at last scattering $\theta \in[0.5,10]$. For the pivot scale we set $k_{s 0}=0.05 \mathrm{Mpc}^{-1}$. When $\mathrm{CMB}$ data are included, we also vary the optical depth to reionization $\tau \in[0.01,0.8]$. We do not consider any massive neutrino contribution. From the parameters above the MCMC code derives the reduced Hubble
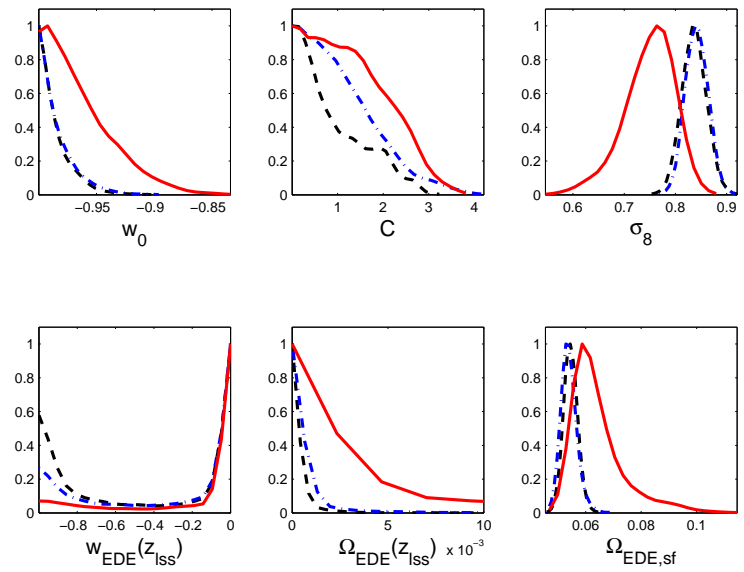

Figure 2: 1D current (marginalized) constraints on the dark energy parameters $w_{0}, C$, as well as $\sigma_{8}$, $w_{\mathrm{EDE}}\left(z_{\mathrm{lss}}\right), \Omega_{\mathrm{EDE}}\left(z_{\mathrm{lss}}\right)$ and $\Omega_{\mathrm{EDE}, \mathrm{sf}}$, from different current data combinations: WMAP $5+\mathrm{BAO}+\mathrm{SN}$ (red solid lines), $\mathrm{WMAP} 5+\mathrm{BAO}+\mathrm{SN}+\mathrm{GRB}+\mathrm{Ly} \alpha$ (blue dash-dot lines) and WMAP $5+\mathrm{BAO}+\mathrm{SN}+\mathrm{GRB}+\mathrm{GFLy} \alpha$ (black dashed lines).

parameter $H_{0}$, the present matter fraction $\Omega_{\mathrm{m} 0}, \sigma_{8}$, and $\Omega_{\mathrm{EDE}, \mathrm{sf}}$ : so, these parameters have non-flat priors and the corresponding bounds must be interpreted with some care. In addition, CosmoMC imposes a weak prior on the Hubble parameter: $h \in[0.4,1.0]$. For part of the analysis, we also present constraints on the growth index $\gamma$, defined as:

$$
\frac{d \ln \delta(a)}{d \ln a}=\Omega_{\mathrm{m}}(a)^{\gamma}
$$

This parameter, introduced by [66], provides a good fit to the growth rate for many different cosmological models and depends on the growth of perturbations, $H(z)$ and $\Omega_{\mathrm{m} 0}$. It has been recently measured by Refs. 67, 68. using mainly the observed growth factors at high and low redshifts and values different from that expected for the pure $\Lambda$ CDM model $(\gamma \sim 0.55)$ could be important for constraining both quintessence models and modified gravity scenarios (see Ref. [69, 70] for forecasting and a more extensive discussion on linear growth rate in modified gravity models).

\section{A. $\mathrm{CMB}+\mathrm{BAO}+\mathrm{SNIa}$}

In Table III we show our main global constraints on the early dark energy model from different current data combinations.

Firstly, we present the constraints from the data combination: $\mathrm{CMB}+\mathrm{BAO}+\mathrm{SNI}$. In Fig. 2 we show the one dimensional distributions of some cosmological parameters from this data combination (solid red lines). For the parameters describing the equation of state of early dark energy, the constraints are still weak, namely the $95 \%$ upper limits are $w_{0}<-0.906$ and $C<2.711$. The limit 
TABLE III. Constraints on the early dark energy model from the current observations. Here we show the mean and the best fit values. For some parameters that are only weakly constrained we quote the $95 \%$ upper limit.

\begin{tabular}{|c|c|c|c|c|c|c|}
\hline \multirow{2}{*}{ Parameter } & WMAP5+BAO+SN & \multicolumn{2}{|c|}{ +GRB+Ly $\alpha$} & \multicolumn{2}{c|}{ +GRB+GFLy $\alpha$} \\
\cline { 2 - 6 } & Mean & BestFit & Mean & BestFit & Mean & BestFit \\
\hline$w_{0}$ & $<-0.906$ & -0.972 & $<-0.952$ & -0.999 & $<-0.958$ & -0.997 \\
$C$ & $<2.711$ & 1.858 & $<2.613$ & 1.628 & $<2.245$ & 0.152 \\
$\Omega_{m}$ & $0.261 \pm 0.014$ & 0.258 & $0.283 \pm 0.014$ & 0.279 & $0.275 \pm 0.014$ & 0.274 \\
$\sigma_{8}$ & $0.748 \pm 0.049$ & 0.734 & $0.842 \pm 0.022$ & 0.863 & $0.836 \pm 0.024$ & 0.846 \\
$\Omega_{\mathrm{EDE}}\left(z_{\text {lss }}\right)$ & $<0.0228$ & 0.0064 & $<0.0029$ & $1.77 \times 10^{-6}$ & $<0.0014$ & $1.71 \times 10^{-9}$ \\
$\Omega_{\mathrm{EDE}, \mathrm{sf}}$ & $0.0643 \pm 0.0076$ & 0.0672 & $0.0540 \pm 0.0029$ & 0.0529 & $0.0546 \pm 0.0024$ & 0.0543 \\
$\gamma$ & - & - & $0.622 \pm 0.139$ & 0.552 & - & - \\
\hline
\end{tabular}

on the present equation of state of dark energy component is still consistent with other recent results in the literature [1, 45]. However, current observational data, could not determine the "running" of equation of state very well. The reason is that the data we use are only the low redshift BAO and SNIa $(z<2)$, as well as the very high redshift $\mathrm{CMB}$ data at last scattering surface $(z \sim 1090)$. Due to the lack of the cosmological probes in the dark ages $(2<z<1100)$, we do not know exactly the evolution of equation of state of early dark energy. Therefore, the constraint on the "running" of equation of state, which is parameterized by $C$, is rather poor.

Consequently, current observations still allow very large amount of early dark energy at high redshift $z \sim$ 1090 as $\Omega_{\mathrm{EDE}}\left(z_{\mathrm{lss}}\right)<0.0228$ (95\% C.L.), which is consistent with the results obtained by Refs. [32, 71]. Early dark energy models with a non-negligible fraction of dark energy density still fit the data very well. Furthermore, there are two peaks in the posterior distribution of equation of state of early dark energy at CMB last scattering surface $w_{\mathrm{EDE}}\left(z_{\mathrm{lss}}\right)$. The first peak around $w_{\mathrm{EDE}}\left(z_{\mathrm{lss}}\right)=0$ is in fact caused by the poor constraint on $C$. When $C$ is larger than one, the equation of state $w_{\mathrm{EDE}}$ will approach zero at high redshift. The larger $C$ is, the faster $w_{\mathrm{EDE}}$ approaches $w=0$ (we will come back to this point in Sect. IV). Considering the weak constraint on $C$, there is significant probability that allows $w_{\mathrm{EDE}}\left(z_{\mathrm{lss}}\right)=0$. The second peak around $w_{\mathrm{EDE}}\left(z_{\mathrm{lss}}\right)=-1$ can be understood easily since the pure $\Lambda \mathrm{CDM}$ model is still favored by the current observations. When $w_{0}$ is close to -1 and $C$ is close to 0 , which is consistent with the data, $w_{\mathrm{EDE}}\left(z_{\mathrm{lss}}\right)$ reaches the cosmological constant value. Therefore, in order to remove the first peak, we need a much more stringent constraint on $C$ from future measurements.

As we showed in Sect. II, early dark energy models also affect structure formation [9]. From Fig. 1] we can see that the linear growth factor of early dark energy model is suppressed significantly by the large value of parameter $C$. The non-negligible dark energy density at high redshifts slows down the linear growth function of the matter perturbation and leads to a low value of $\sigma_{8}$ today. Using $\mathrm{CMB}+\mathrm{BAO}+\mathrm{SNIa}$ data combination, illustrated in Fig. 2, we obtain the limit on $\sigma_{8}$ today of $\sigma_{8}=0.748 \pm 0.049$ (68\% C.L.), which is obviously lower than one obtained in the pure $\Lambda \mathrm{CDM}$ framework:
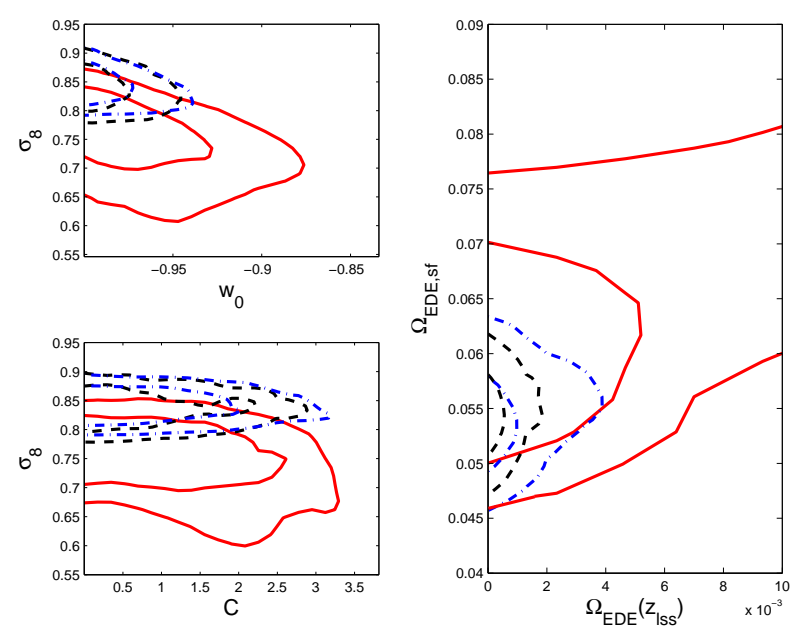

Figure 3: 2D (marginalized) contours of some parameters from different current data combinations: WMAP5+BAO+SN (red solid lines), $\mathrm{WMAP} 5+\mathrm{BAO}+\mathrm{SN}+\mathrm{GRB}+\mathrm{Ly} \alpha$ (blue dash-dot lines) and WMAP5+BAO+SN+GRB+GFLy $\alpha$ (black dashed lines).

$\sigma_{8}=0.812 \pm 0.026$ [1], while the error bar is enlarged by a factor of two. Therefore, the $\sigma_{8}$ today and $C$ are anti-correlated, as shown in Fig. 3. On the other hand, the $\sigma_{8}$ and equation of state $w_{0}$ at present also have an anti-correlation. When $w_{0}$ is increased, the fraction of dark energy density becomes large, which also leads the lower $\sigma_{8}$ today.

Finally, we discuss the average value of the fraction of dark energy in the structure formation era $\Omega_{\mathrm{EDE}, \mathrm{sf}}$. In Table III we can find that the current constraint is $\Omega_{\mathrm{EDE}, \mathrm{sf}}=0.0643 \pm 0.0076$ at $1 \sigma$ confidence level. From Eq.(3) and Fig. 3] it is easy to see that a large $\Omega_{\mathrm{EDE}}\left(z_{\mathrm{lss}}\right)$ leads to the high $\Omega_{\mathrm{EDE}, \mathrm{sf}}$. In Fig. 4 we also plot the two dimensional plots between $\Omega_{\mathrm{EDE}, \mathrm{sf}}$ and other related parameters. As we discussed before, when $w_{0}$ and $C$ become large, the fraction of dark energy density at high redshifts will also be large. On the other hand, when $\Omega_{\mathrm{EDE}, \mathrm{sf}}$ increases, the linear growth function of matter perturbation will be slowed down and the value of $\sigma_{8}$ today will decrease consequently: $\Omega_{\mathrm{EDE}, \mathrm{sf}}$ and the $\sigma_{8}$ 

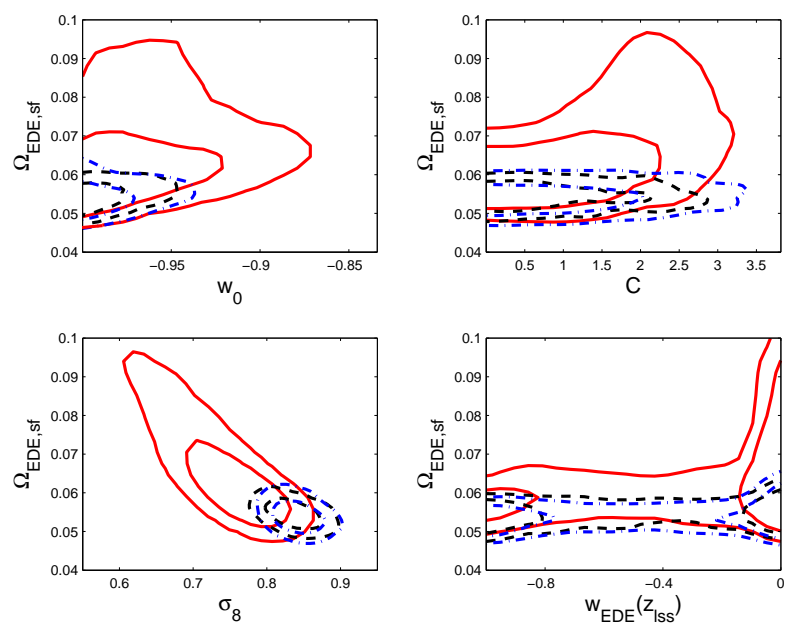

Figure 4: $2 \mathrm{D}$ (marginalized) contours of $\Omega_{\mathrm{EDE}, \mathrm{sf}}$ versus $w_{0}, C, \sigma_{8}$ and $w_{\mathrm{EDE}}\left(z_{\mathrm{lss}}\right)$ from different current data combinations: WMAP5+BAO+SN (red solid lines), $\mathrm{WMAP} 5+\mathrm{BAO}+\mathrm{SN}+\mathrm{GRB}+\mathrm{Ly} \alpha$ (blue dash-dot lines) and WMAP5+BAO+SN+GRB+GFLy $\alpha$ (black dashed lines).

today are then obviously anti-correlated.

To sum up, using CMB, BAO and SNIa, we constrain the amount of dark energy at the last scattering surface to be less than $2 \%$ and in the structure formation era to be of the order of $6 \%$. This last number is in rough agreement with the results of [72], who used WMAP 3 year data and some external data sets, although their values are somewhat smaller $(\sim 4 \%)$ and this is due to the slightly different definition of $\Omega_{\mathrm{EDE}, \mathrm{sf}}$.

\section{B. Adding High-Redshift Probes}

From the results above, we can see that without observations in or around the so called dark ages, the constraints on the early dark energy model are interesting but not very tight. In this subsection we add some high-redshift observational data, such as GRBs, Ly $\alpha$ and GFLy $\alpha$ data, to improve our constraints. Because most of GFLy $\alpha$ data are obtained from the Ly $\alpha$ forest power spectrum, in order to avoid overestimating the weight of Ly $\alpha$ data, in our analysis we do not use GFLy $\alpha$ and Ly $\alpha$ data together. And the results, as we expect, from these two data combinations are consistent between each other. Therefore, in the following we mainly present the global fitting results obtained from those that include GRB and GFLy $\alpha$ data.

When adding the high-redshift probes, the constraints on $w_{0}$ and $C$ improve significantly: $w_{0}<-0.958$ and $C<2.245$ at $95 \%$ confidence level, while their best fit values are very close to the pure $\Lambda$ CDM model. Naturally, the $95 \%$ upper limit of $\Omega_{\mathrm{EDE}}\left(z_{\mathrm{lss}}\right)$ has also been tightened by a factor of ten, $\Omega_{\mathrm{EDE}}\left(z_{\mathrm{lss}}\right)<1.4 \times 10^{-3}$. A large number of early dark energy models have been ruled out by the high-redshift probes. The high-redshift observations are indeed effective in constraining further the early dark energy models investigated here. Note that adding only the power spectrum information as provided by Ly $\alpha$ improves the constraints on $\Omega_{\mathrm{EDE}}\left(z_{\mathrm{lss}}\right)$ by a factor five, when compared to $\mathrm{CMB}+\mathrm{BAO}+\mathrm{SNIa}$, so the growth factor information is really fundamental in constraining the EDE model. However, the double peak in the distribution of $w_{\mathrm{EDE}}\left(z_{\mathrm{lss}}\right)$ is still present, which implies that the current data are not accurate enough to measure $w_{\mathrm{EDE}}\left(z_{\mathrm{lss}}\right)$, even when high-redshift probes are added.

Another effect of adding high-redshift probes is the larger value of $\sigma_{8}$ today. As we know, the $\operatorname{Ly} \alpha$ forest data favor a larger $\sigma_{8} \sim 0.9$ than the CMB measurement $^{3}$. When we add the GFLy $\alpha$ or Ly $\alpha$ forest data, we obtain a higher $\sigma_{8}$ today than before: $\sigma_{8}=0.836 \pm 0.024$ $(1 \sigma)$. However, this is still smaller than the value favored by Ly $\alpha$ forest data alone in the pure $\Lambda$ CDM model and consistent with WMAP $5+\mathrm{BAO}+\mathrm{SN}$ measurement of [1] . We also note that if we use only VHSLy $\alpha$ data sets and adding them to WMAP $5+\mathrm{BAO}+\mathrm{SN}$ and GRBs the results do not change significantly as compared to having SDSSLy $\alpha$ and VHSLy $\alpha$ together. This is mainly due to the constraining power of the growth factors measurement obtained with VHSLya.

The conclusion of this section is that adding higher redshift probes constrains the energy density of dark energy at the last scattering surface to be around $0.1 \%$.

\section{Growth Index $\gamma$}

Finally, we extend our discussion by briefly addressing the perspective of constraining the linear growth index $\gamma$. Measurements of the growth history of cosmic structures combine information on both cosmic expansion and the underlying theory of gravity, the parameter $\gamma$ is thus a unique prediction of any modified gravity model and a simultaneous fitting of this parameter and other dynamical probes could provide constraints on modified gravity scenarios and dynamical dark energy models.

By using all the current data available we obtain $\gamma=0.622 \pm 0.139(1 \sigma)$ error bar for our EDE model, which is in agreement with the $\Lambda$ CDM and with the values obtained by Refs. 67, 68] (with slightly smaller error bars). The present error on $\gamma$ is also similar to that inferred from future weak lensing and SNIa data by Refs. [69].

\footnotetext{
3 This fact does not imply a contradiction or a failure of the $\Lambda$ CDM model but there is indeed a tension between small scale constraints and the large scale ones. However, more recent studies that combine WMAP year 3 , the Lyman- $\alpha$ forest and weaklensing measurements of the COSMOS-z survey 24] point to a value of $\sigma_{8}=0.800 \pm 0.023$, which is in good agreement with the findings of WMAP year 5 plus BAO and SNe [1].
} 

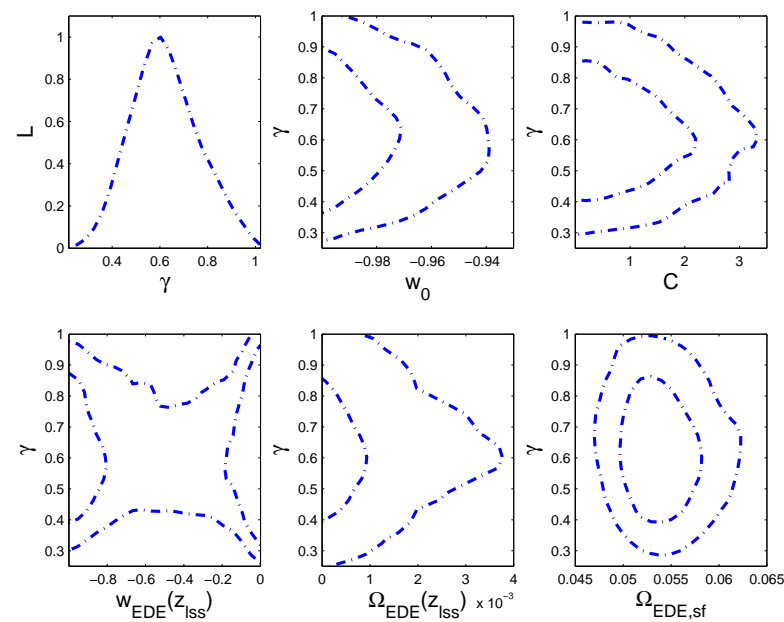

Figure 5: The posterior distribution of $\gamma$ and 2D (marginalized) contours of $\gamma$ and $w_{0}, C, w_{\mathrm{EDE}}\left(z_{\mathrm{lss}}\right), \Omega_{\mathrm{EDE}}\left(z_{\mathrm{lss}}\right)$ and $\Omega_{\mathrm{EDE}, \text { sf }}$ from WMAP $5+\mathrm{BAO}+\mathrm{SN}+\mathrm{GRB}+\mathrm{Ly} \alpha$ (blue dotted lines).

TABLE IV. Constraints on the early dark energy model

from future measurements. We also list the standard deviation of these parameters based on the future mock data sets. For parameters that are only weakly constrained we quote the $95 \%$ upper limit instead.

\begin{tabular}{|c|c|c|}
\hline Parameter & Fiducial $\Lambda$ CDM & Fiducial EDE \\
\hline$w_{0}$ & $<-0.975$ & {$[-0.985,-0.920]$} \\
$C$ & $<2.635$ & {$[0.256,2.300]$} \\
$\Omega_{m}$ & 0.0029 & 0.0032 \\
$\sigma_{8}$ & 0.0078 & 0.0145 \\
$\Omega_{\mathrm{EDE}}\left(z_{\mathrm{lss}}\right)$ & $<1.02 \times 10^{-4}$ & $<0.011$ \\
$\Omega_{\mathrm{EDE}, \mathrm{sf}}$ & 0.0011 & 0.0037 \\
$\gamma$ & 0.026 & 0.048 \\
\hline
\end{tabular}

In Figure 5 we show the 2-dimensional contours of $\gamma$ vs. $\sigma_{8}, C, w_{0}$ and $\Omega_{\mathrm{EDE}}\left(z_{\mathrm{lss}}\right)$ to address possible degeneracies of this parameter. For the current data sets, we note that the degeneracies are weak and only the degeneracy in the $\gamma-\Omega_{\mathrm{EDE}, \text { sf }}$ plane is significant. This is easily understood since a larger $\gamma$ implies a faster growth of structures and thereby a smaller amount of dark energy.

\section{RESULTS FROM FUTURE DATA SETS}

Since the present data do not give very stringent constraints on the parameters of early dark energy model, it is worthwhile discussing whether future data could determine these parameters conclusively. For this purpose we have performed a further analysis and we have chosen two fiducial models in perfect agreement with current data: a pure $\Lambda \mathrm{CDM}$ model and an EDE model with parameters taken to be the best-fit values of Table III from the current constraints of $\mathrm{CMB}+\mathrm{BAO}+\mathrm{SNI}$. This latter model will be labelled EDE1, and its evolution is shown
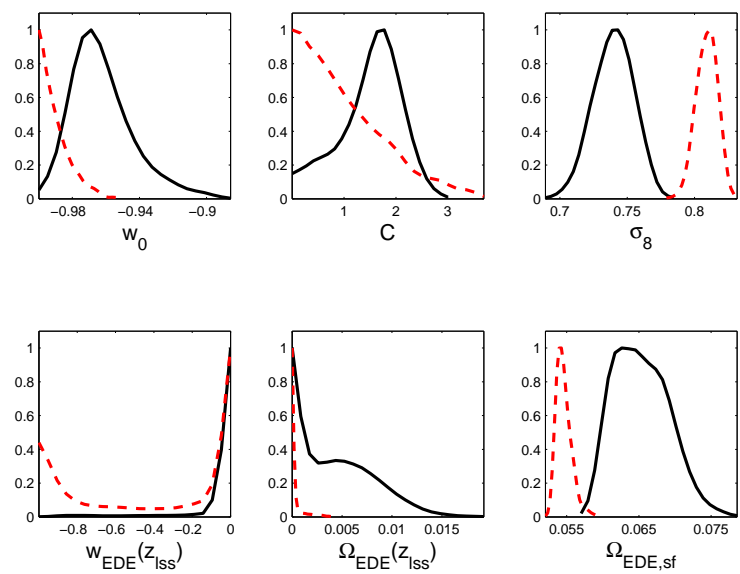

Figure 6: 1D current (marginalized) constraints on the dark energy parameters $w_{0}, C$, as well as $\sigma_{8}, w_{\mathrm{EDE}}\left(z_{\mathrm{lss}}\right), \Omega_{\mathrm{EDE}}\left(z_{\mathrm{lss}}\right)$ and $\Omega_{\mathrm{EDE}, \mathrm{sf}}$ from future measurements with the fiducial models: $\Lambda$ CDM (red dashed lines), EDE1 model (black solid lines).

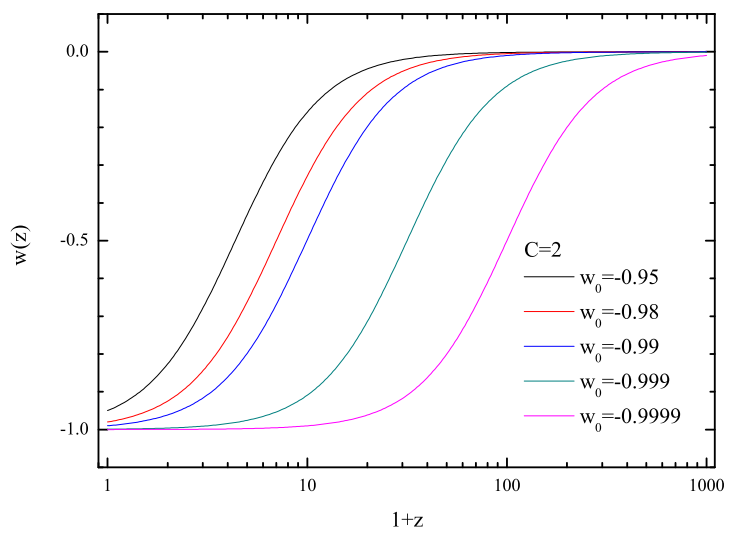

Figure 7: Evolution of different equation of state for the Mocker model when fixing $C=2$. All these models fit low redshift $(z<2)$ and $\mathrm{CMB}$ constraints but have very different evolution at intermediate redshifts.

in Fig⿴囗十

\section{A. Fiducial $\Lambda \mathrm{CDM}$}

Firstly, we choose the pure $\Lambda \mathrm{CDM}$ as the fiducial model. In Table IV we list the forecasts for some parameters using all the future measurements described in Section IIB2. GRBs, SNIa, Ly $\alpha$ (matter power spectrum and growth factors) and the AP test.

Due to the smaller error bars of the mock data sets, the constraint on $w_{0}$ improves significantly, namely the $95 \%$ upper limit is now $w_{0}<-0.975$. The fraction of 


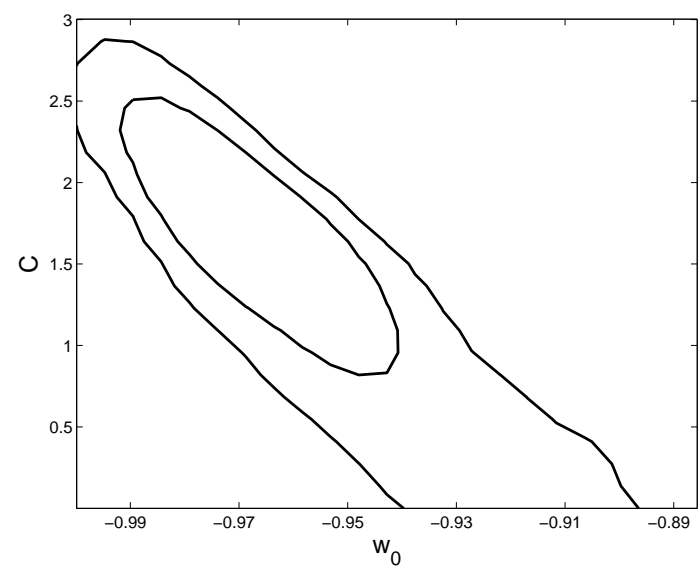

Figure 8: 2D marginalized contours $C$ vs $w_{0}$ from future mock measurements of the fiducial EDE1 model.

early dark energy density has been limited very stringent, $\Omega_{\mathrm{EDE}}\left(z_{\mathrm{lss}}\right)<1.02 \times 10^{-4}$ (95\% C.L.), which is reduced by another factor of ten, compared with the present constraint. The error on the $\gamma$ parameter that we forecast is 0.026 , while the error on the amount of EDE in the structure formation era is about $1.1 \times 10^{-3}$. Therefore, we can foresee a very precise determination of the amount of dark energy in the dark ages (or in the structure formation era) with this future data set.

By contrast, the $95 \%$ upper limit on $C$ has not been improved, $C<2.635$. This is due to the chosen parameterization of early dark energy. In Fig. 7 we illustrate the different evolution of several EDE equations of state varying $w_{0}$ and keeping $C=2$. We note that the smaller the redshift at which $w_{0}$ approaches the cosmological constant, the smaller the redshift at which $w(z)$ flattens to zero in the high redshift universe. For example, if $w_{0}=-0.95$, we have $w(z=10) \sim-0.1$ and $C=2$ has been ruled out obviously. On the other hand, if $w_{0}=-0.9999$, we still have $w(z=10) \sim-1$. Consequently, $C=2$ is still favored by the data. Therefore, even if the constraint on $w_{0}$ becomes stringent, a large value of $C$ is still allowed, and then the constraint on $C$ will be somewhat poorer. Moreover, due to the allowed large value of $C$, the first peak of $w_{\mathrm{EDE}}\left(z_{\mathrm{lss}}\right)$ around $w=0$ is still present, as shown in Fig. 6. where the 1D marginalized constraints at the last scattering surface are shown.

\section{B. Fiducial EDE}

For our EDE fiducial model we choose the best fit values of current constraint as the fiducial model to simulate the future measurements, $w_{0}=-0.972$ and $C=1.858$ (EDE1).

In Fig. 6, we plot the one dimensional distributions of $w_{0}$ and $C$. The $95 \%$ confidence level is $-0.985<$ $w_{0}<-0.920$ and $0.256<C<2.300$, respectively. These results imply that the future measurements could distinguish the pure $\Lambda \mathrm{CDM}$ model and early dark energy models at least at $5 \sigma$ confidence level. We also show the contour in the $\left(w_{0}, C\right)$ plane in Fig. 8 . We note that even with these futuristic data sets simulated in a conservative way the value $C=0$ is still allowed at a $2 \sigma$ confidence level. When $w_{0}$ increases, $C$ must decrease in order to match the current observations. Thus, $w_{0}$ and $C$ are anti-correlated. Consequently, the allowed value of $\Omega_{\mathrm{EDE}}\left(z_{\mathrm{lss}}\right)$ could be very large: $\Omega_{\mathrm{EDE}}\left(z_{\mathrm{lss}}\right)<1.1 \times 10^{-2}$ (95\% C.L.). Furthermore, with this fiducial model, the double peak in the distribution of $w_{\mathrm{EDE}}\left(z_{\mathrm{lss}}\right)$ disappears: because the pure $\Lambda \mathrm{CDM}$ is disfavored at more than $5 \sigma$ confidence level.

In Fig. 6 we clearly appreciate the very distinctive predictions of $\Omega_{\mathrm{EDE} \text {,sf }}$ and $\sigma_{8}$ for the $\Lambda \mathrm{CDM}$ and EDE1 models. The values obtained for $\Omega_{\mathrm{EDE}, \mathrm{sf}}$ and $\sigma_{8}$ are different by $20 \%$ and $10 \%$, respectively, and can be measured at very high precision in the two cases.

We are also interested in understanding which of the different cosmological probes give the most stringent constraints. In order to investigate this we perform three different runs: we find that the best constraints are obtained when future $\operatorname{Ly} \alpha$ and GFLy $\alpha$ are added to Planck and SNAP data, while adding AP only to Planck and SNAP results in the the worst constraints. In the third run, we consider adding only GRBs mock data to Planck and SNAP and in this case the results are in between those inferred from Ly $\alpha$ and AP test. The degeneracies between different parameters are similar in three cases. We also checked that degrading the accuracy of the AP test to $10 \%$ as opposed to the chosen $5 \%$ has not a significant impact on the recovered parameters.

We also consider the results in terms of $\gamma$ has done in Section IIC We find that we could constrain $\gamma$ to high significance in the two cases: the standard deviations are 0.026 and 0.048 , which are reduced by a factor of five and three, when compared to the current constraints, respectively. By looking at Fig. 9, we can now see that the constraints on $\gamma$ are more precise and degeneracies with $\Omega_{\mathrm{EDE}}\left(z_{\mathrm{lss}}\right)$ and $\Omega_{\mathrm{EDE}, \mathrm{sf}}$ are stronger.

\section{CONCLUSIONS AND DISCUSSION}

With the availability of new data from SNIa, and the advent of relatively new cosmological probes, such as Ly $\alpha$ and GRBs in the high redshift universe, there has been an increasing interest in the study of dynamical dark energy models with a significant energy contribution in the structure formation era. In this paper we present constraints on a particular early dark energy model using the latest observations. We use the Mocker parameterization proposed by Ref.[32], but our results are general since the amount of dark energy at the last scattering surface and in the structure formation era have also been quoted in order to better compare with other possible dark energy parameterizations [72]. 

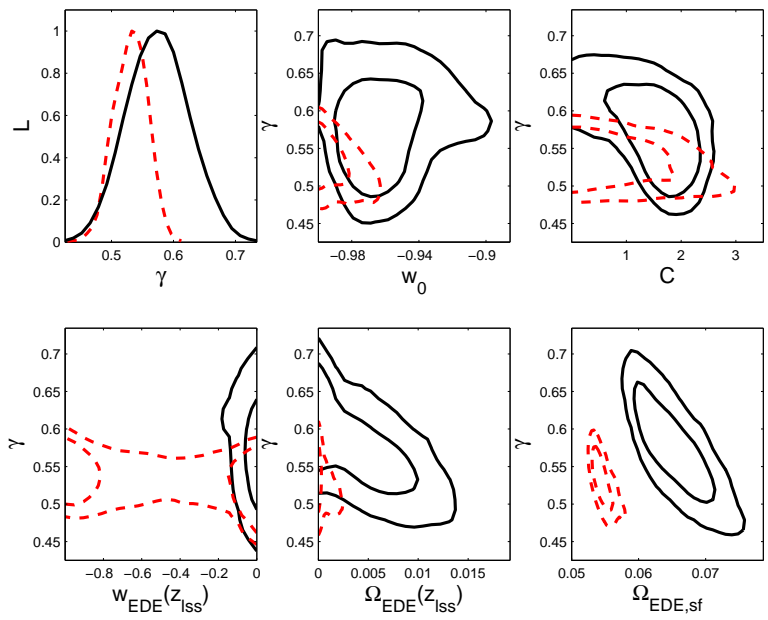

Figure 9: The posterior distribution of $\gamma$ and 2D (marginalized) contours of $\gamma$ and $w_{0}, C, w_{\mathrm{EDE}}\left(z_{\mathrm{lss}}\right), \Omega_{\mathrm{EDE}}\left(z_{\mathrm{lss}}\right)$ and $\Omega_{\mathrm{EDE}, \mathrm{sf}}$ from future measurements with the fiducial models: $\Lambda \mathrm{CDM}$ (red dashed lines), EDE1 model (black solid lines).

We find that current constraints based on CMB, BAO and SNIa data limit the amount of dark energy density at the last scattering surface to be $2 \%$, while in the structure formation period is of the order of $6 \%$. If we add high redshift GRBs and the Ly $\alpha$ forest data, in the form of a matter power spectrum or in the form of a growth factor measurement, the constraints improve by a factor five and fifteen, respectively. The $\Lambda \mathrm{CDM}$ model is still a very good fit to the data currently available and only upper limits on the amount of dark energy at the last scattering could be quoted.

We also forecast what could be done with future data sets in particular: Planck for the CMB; GRBs and SNIa luminosity distances as could be measured by SNAP; a better measurement of Ly $\alpha$ matter power spectrum and growth factors as could be obtained from SDSS-III and the AP test performed on $\mathrm{HI} 21 \mathrm{~cm}$ maps from LOFAR. With these futuristic mock data sets we simulate two different fiducial models that are currently in agreement with observations a $\Lambda$ CDM model and an EDE model with about $6 \%$ contribution to the energy density in the structure formation epoch. We find that the two models will be clearly distinguishable at more than $5 \sigma$ confidence level. Thereby, the role of these high redshift probes is crucial in discriminating between pure $\Lambda \mathrm{CDM}$ and quintessence and/or modified gravity models.

Furthermore, motivated by the fact that the growth factors at high redshift seem to have the highest constraining power, we also address the perspectives of measuring the parameter $\gamma$ that parameterizes the growth rate of structures and is usually a very precise predic- tion for any quintessence and/or modified gravity model. Even in this case, we find that in the two models we could constrain $\gamma$ to high significance: the standard deviations are reduced by a factor of five and three, when compared with the current constraint, respectively.

We conclude this Section with some words of caution: while high redshift probes are important in constraining early dark energy models, the obtained values from present and future constraints rely mainly on the Ly $\alpha$ forest and its derived growth factors. These measurements, summarized partly in Table I, have been obtained in the framework of pure $\Lambda \mathrm{CDM}$ model and are valid only when small deviations from this model are considered. We believe that we are in such a situation here, where the amount of dark energy has little impact on observed $\operatorname{Ly} \alpha$ quantities and this effect is possibly degenerate with other parameters that have been already marginalized over to obtain the final estimate. However, further work using both numerical hydrodynamical simulations as long with an accurate control of systematics should be done in order to better check this (see for example Ref. [73]). Along the same lines, while the role of SNIa as cosmological probes is somewhat reasonably well established, those of GRBs is still controversial. We tried to circumvent this problem by marginalizing over the nuisance parameters that enter the calibration of the GRBs distance moduli but, even in this case, more observational and theoretical work is needed in order to trust quantitatively the results. Even regarding the forecasting using the AP test, we tried to implement these constraints in a conservative way, in a range of redshift that could be observed by LOFAR, without assuming that a full matter power spectrum could be measured from the observed brightness temperature (this of course will have a fundamental impact especially at even higher redshifts than those considered here).

However, even considering these cautious remarks, our work clearly emphasizes the capabilities of present and future data in an intermediate redshift range which is poorly probed by the observations, to quantitatively constrain the dark energy component and its evolution. We consider these observations as very important since they can open up a completely new window on observational cosmology and high energy physics and provide new insights on either modified gravity or quintessence scenarios.

\section{ACKNOWLEDGMENTS}

The numerical analysis has been performed on the Shanghai Supercomputer Center (SSC). We thank Viviana Acquaviva for useful discussions. 
[3] S. Weinberg, Rev. Mod. Phys. 61, 1 (1989); I. Zlatev, L. M. Wang and P. J. Steinhardt, Phys. Rev. Lett. 82, 896 (1999).

[4] R. D. Peccei, J. Sola and C. Wetterich, Phys. Lett. B 195, 183 (1987); C. Wetterich, Nucl. Phys. B 302, 668 (1988); B. Ratra and P. J. E. Peebles, Phys. Rev. D 37, 3406 (1988); P. J. E. Peebles and B. Ratra, Astrophys. J. 325, L17 (1988).

[5] R. R. Caldwell, Phys. Lett. B 545, 23 (2002).

[6] T. Chiba, T. Okabe and M. Yamaguchi, Phys. Rev. D 62, 023511 (2000); C. Armendariz-Picon, V. F. Mukhanov and P. J. Steinhardt, Phys. Rev. Lett. 85, 4438 (2000).

[7] B. Feng, X. L. Wang and X. Zhang, Phys. Lett. B 607, 35 (2005).

[8] E. J. Copeland, M. Sami and S. Tsujikawa, Int. J. Mod. Phys. D 15, 1753 (2006).

[9] M. Doran, J. M. Schwindt and C. Wetterich, Phys. Rev. D 64, 123520 (2001).

[10] E. V. Linder and A. Jenkins, Mon. Not. Roy. Astron. Soc. 346, 573 (2003).

[11] K. Dolag, M. Bartelmann, F. Perrotta, C. Baccigalupi, L. Moscardini, M. Meneghetti and G. Tormen, Astron. Astrophys. 416, 853 (2004).

[12] R. Mainini, A. V. Maccio, S. A. Bonometto and A. Klypin, Astrophys. J. 599, 24 (2003).

[13] D. Crociani, M. Viel, L. Moscardini, M. Bartelmann and M. Meneghetti, Mon. Not. Roy. Astron. Soc. 385, 728 (2008).

[14] M. Bartelmann, M. Doran and C. Wetterich, Astron. Astrophys. 475, 27 (2006).

[15] C. Fedeli and M. Bartelmann, Astron. Astrophys. 461, 49 (2007).

[16] M. Manera and D. F. Mota, Mon. Not. Roy. Astron. Soc. 371, 1373 (2006); M. J. Francis, G. F. Lewis and E. V. Linder, arXiv:0810.0039 D. F. Mota, JCAP 0809, 006 (2008).

[17] M. Grossi and V. Springel, arXiv:0809.3404.

[18] N. Padmanabhan et al., Mon. Not. Roy. Astron. Soc. 378, 852 (2007).

[19] D. J. Eisenstein et al. Astrophys. J. 633, 560 (2005).

[20] S. Cole et al. Mon. Not. Roy. Astron. Soc. 362, 505 (2005).

[21] Y. Gong, T. J. Zhang, T. Lan and X. L. Chen, arXiv:0810.3572, M. Kilbinger et al., arXiv:0810.5129.

[22] H. Li et al., arXiv:0812.1672.

[23] A. G. Riess et al., Astrophys. J. 659, 98 (2007).

[24] J. Lesgourgues, M. Viel, M. G. Haehnelt and R. Massey, JCAP 0711, 008 (2007).

[25] P. McDonald et al., Astrophys. J. 635, 761 (2005).

[26] B. E. Schaefer, Astrophys. J. 660, 16 (2007).

[27] H. Li, M. Su, Z. Fan, Z. Dai and X. Zhang, Phys. Lett. B 658, 95 (2008).

[28] A. Vikhlinin et al., arXiv:0812.2720.

[29] C. Alcock and B. Paczyński, Nature 281, 358 (1979).

[30] A. Nusser, Mon. Not. Roy. Astron. Soc. 364, 743 (2005).

[31] R. Barkana, Mon. Not. Roy. Astron. Soc. 372, 259 (2006).

[32] E. V. Linder, Astropart. Phys. 26, 16 (2006).

[33] E. V. Linder and G. Robbers, JCAP 0806, 004 (2008).

[34] M. Doran, S. Stern and E. Thommes, JCAP 0704, 015 (2007).

[35] C. Wetterich, Phys. Lett. B 594, 17 (2004).

[36] A. Lewis and S. Bridle, Phys. Rev. D 66, 103511 (2002); URL: http://cosmologist.info/cosmomc/
[37] J. Weller and A. M. Lewis, Mon. Not. Roy. Astron. Soc. 346, 987 (2003); G. B. Zhao, J. Q. Xia, M. Li, B. Feng and X. Zhang, Phys. Rev. D 72, 123515 (2005); J. Q. Xia, G. B. Zhao, B. Feng, H. Li and X. Zhang, Phys. Rev. D 73, 063521 (2006).

[38] J. Dunkley et al., arXiv:0803.0586, E. L. Wright et al., arXiv:0803.0577. M. R. Nolta et al., arXiv:0803.0593. B. Gold et al., arXiv:0803.0715 G. Hinshaw et al., arXiv:0803.0732 URL: http://lambda.gsfc.nasa.gov/

[39] G. Huetsi, Astron. Astrophys. 449, 891 (2006).

[40] W. J. Percival et al., Mon. Not. Roy. Astron. Soc. 381, 1053 (2007).

[41] A. Albrecht et al., arXiv:astro-ph/0609591

[42] T. Okumura, T. Matsubara, D. J. Eisenstein, I. Kayo, C. Hikage, A. S. Szalay and D. P. Schneider, Astrophys. J. 677, 889 (2008).

[43] D. J. Eisenstein and W. Hu, Astrophys. J. 496, 605 (1998).

[44] E. Di Pietro and J. F. Claeskens, Mon. Not. Roy. Astron. Soc. 341, 1299 (2003).

[45] J. Q. Xia, H. Li, G. B. Zhao and X. Zhang, Phys. Rev. D 78, 083524 (2008).

[46] W. L. Freedman et al., Astrophys. J. 553, 47 (2001).

[47] H. Li, J. Q. Xia, J. Liu, G. B. Zhao, Z. H. Fan and X. Zhang, Astrophys. J. 680, 92 (2008).

[48] C. Firmani, V. Avila-Reese, G. Ghisellini and G. Ghirlanda, Rev. Mex. Astron. Astrofis. 43, 203 (2007).

[49] M. Viel, M. G. Haehnelt and V. Springel, Mon. Not. Roy. Astron. Soc. 354, 684 (2004).

[50] M. Viel and M. G. Haehnelt, Mon. Not. Roy. Astron. Soc. 365, 231 (2006).

[51] E. Hawkins et al., Mon. Not. Roy. Astron. Soc. 346, 78 (2003); L. Verde et al., Mon. Not. Roy. Astron. Soc. 335, 432 (2002).

[52] T. S. Kim, M. Viel, M. G. Haehnelt, R. F. Carswell and S. Cristiani, Mon. Not. Roy. Astron. Soc. 347, 355 (2004).

[53] R. A. C. Croft et al., Astrophys. J. 581, 20 (2002).

[54] P. McDonald et al., Astrophys. J. Suppl. 163, 80 (2006).

[55] Planck Collaboration, arXiv:astro-ph/0604069.

[56] J. Q. Xia, H. Li, G. B. Zhao and X. Zhang, Int. J. Mod. Phys. D 17, 2025 (2008).

[57] URL: http://snap.lbl.gov/

[58] A. G. Kim, E. V. Linder, R. Miquel and N. Mostek, Mon. Not. Roy. Astron. Soc. 347, 909 (2004).

[59] D. Hooper and S. Dodelson, Astropart. Phys. 27, 113 (2007).

[60] URL: http://heasarc.gsfc.nasa.gov/docs/swift/swiftsc.html

[61] URL: http://www.eso.org/instruments/xshooter/index.html/

[62] S. Gratton, A. Lewis and G. Efstathiou, Phys. Rev. D 77, 083507 (2008).

[63] URL: http://www.lofar.org/

[64] S. Wyithe, A. Loeb and P. Geil, Mon. Not. Roy. Astron. Soc. 383, 1195 (2008).

[65] URL: http://www.skatelescope.org/

[66] P. J. E. Peebles, Large-Scale Structure of the Universe, (Princeton U. Press: 1980); L. M. Wang and P. J. Steinhardt, Astrophys. J. 508, 483 (1998).

[67] S. Nesseris and L. Perivolaropoulos, Phys. Rev. D 77, 023504 (2008).

[68] C. Di Porto and L. Amendola, Phys. Rev. D 77, 083508 (2008).

[69] D. Huterer and E. V. Linder, Phys. Rev. D 75, 023519 
(2007); E. V. Linder and R. N. Cahn, Astropart. Phys. 28, 481, (2007).

[70] G. B. Zhao, L. Pogosian, A. Silvestri and J. Zylberberg, arXiv:0809.3791; V. Acquaviva, A. Hajian, D. N. Spergel and S. Das, Phys. Rev. D 78, 043514 (2008).

[71] W. Lee and K. W. Ng, Phys. Rev. D 67, 107302 (2003).
[72] M. Doran and G. Robbers, JCAP 0606, 026 (2006).

[73] M. Viel, S. Matarrese, T. Theuns, D. Munshi and Y. Wang, Mon. Not. Roy. Astron. Soc. 340, L47 (2003); P. McDonald and D. J. Eisenstein, Phys. Rev. D 76, 063009 (2007). 